\title{
COMUNICACIÓN
}

\section{EVALUACIÓN DE LOS PARÁMETROS PRODUCTIVOS DE CODORNICES, VARIEDAD JAPONESA (Coturnix coturnix japonica) PROVENIENTES DE TRES PLANTELES REPRODUCTORES DE LIMA}

\author{
Patricia Sulca A.+, Edgardo Figueroa T. ${ }^{1}$ y Fernando Carcelén $C .^{2}$
}

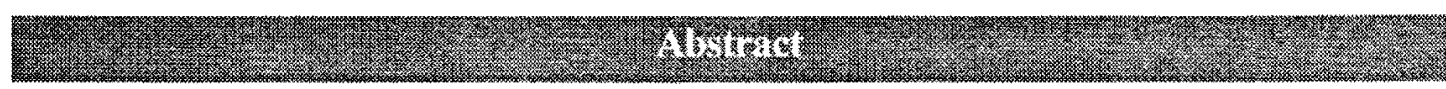

The productive parameters of live weight, feed intake, feed conversion and mortality, during the first four weeks of life in Japanese quails (Coturnix coiurnix japonica) are reported. Three breeding groups (L1, L2 and L3) consisting of 1,400 birds each, were subdivided into 14 random experimental units of 100 caged quail. Statistically significant differences $(P<0.05)$ were found in final live weight $114.3 \mathrm{~g}, 113.8 \mathrm{~g}$ and $108.6 \mathrm{~g}$ for groups $\mathrm{LI}, \mathrm{L} 2$ and L3, as well as for final weight gain $(106.2 \mathrm{~g}, 105.5 \mathrm{~g}$ and $100.4 \mathrm{~g})$. Differences in cumulative feed consumption were statistically significant $(\mathrm{P}<0.05)$ between group L1 (309.3g) and L3 (305.6g), as well as between L2 (309.1 g) and L3 (305.6g), but not for groups L1 and L2. Feed conversion rates of 2.71 (group L1), 2.72 (group L2) and 2.8 (group L3) were not statistically significant except for group L3 ( $>0.05$ ). Mortality rates of $3.4 \%, 4.1 \%$ and $3.2 \%$ for $\mathrm{L} 1, \mathrm{~L} 2$ and $\mathrm{L} 3$ respectively, were not statistically different $(\mathrm{P}>0.05)$.

Key words: Japanese quail, live weight, feed intake, feed conversion, mortality

Palabras clave: Codomiz japonesa, peso vivo, consumo de alimento, conversión alimenticia, mortalidad.

La crianza de codornices en el país se viene desarrollando en respuesta a la creciente demanda por sus productos: huevo y carne. La experiencia es aún incipiente entre nuestros criadores y de allí la importancia de contribuir con información que permita lograr un cuerpo de conocimientos útiles sobre esta actividad. Este trabajo tiene como objetivo evaluar parámetros productivos como peso vivo, ganancia de peso, consumo de alimento,

$\dagger$ Este articulo fue presentado para su publicación por la Dra Patricia Sulca A. recientemente fallecida. Sirva el mismo como testimonio de su brillante trabajo y compromiso con la Facultad de Medicina Veterinaria de la UNMSM.

'UNMSM-FMV-Laboratorio de Patología Aviar y Producción Avicola. E-mail: efigueroa_1999@yahoo.com.

${ }^{2}$ UNMSM-FMV-Laboratorio de Bioquimica, Nutrición y Alimentación Animal. conversión alimenticia y mortalidad en codornices hasta la cuarta semana de edad.

Se emplearon 4200 codornices de la variedad japonesa divididas en 3 lotes de 1 400 cada uno, de un día de edad, sin sexar, procedentes de UNMSM (L1); UNA, La Molina (L2) y granjita, La Molina (L3). Cada Lote según su procedencia fue dividido en 14 subgrupos (o unidades experimentales) de 100 aves cada uno. El manejo fue igual para los tres lotes y durante las cuatro semanas de crianza las codornices recibieron ad libitum alimento comercial con un contenido de $26 \%$ de proteína cruda y $2.8 \mathrm{Mcal} /$ $\mathrm{kg}$ de alimento.

Se obtuvo el peso vivo grupal de las 100 codomices de cada unidad experimental, al finalizar cada semana de crianza y durante 
cuatro semanas para obtener la ganancia de peso semanal y la ganancia de peso acumulada por ave; así mismo se estimó cada semana (CAS) y de manera acumulada (CAA) el consumo de alimento aplicando la siguiente fórmula:

\section{CAS o CAA por ave, $g=$}

\section{Alimento suministrado - Alimento sobrante

$$
N^{\circ} \text { Aves }
$$

El índice de conversión alimenticio (ICA) se evaluó para cada grupo de 100 aves. Estos valores se obtuvieron de acuerdo a las fórmulas siguientes:

$$
\text { ICA semanal }=\frac{\text { Alimento consumido }}{\text { Ganancia de peso }}
$$

ICA acumulado por ave $=$

\section{Alimento consumido Peso vivo}

Se llevó el control semanal de mortalidad y el diagnóstico de la causa de muerte se efectuó mediante la necropsia de cada ave.
El porcentaje de mortalidad semanal se obtuvo mediante la fórmula siguiente:

Mortalidad $(\%)=$

$$
\frac{N^{\circ} \text { de aves muertas }}{N^{\circ} \text { de aves vivas iniciales }} \times 100
$$

El diseño experimental utilizado fue el irrestricto al azar y los datos productivos obtenidos durante las cuatro semanas de crianza, fueron analizados mediante el análisis de varianza de una sola vía y las diferencias entre medias se realizó mediante la prueba de comparación múltiple de DMS de Fisher (protegida).

En el Cuadro 1, se muestra los pesos, ganancia de peso, consumo de alimento, índice de conversión alimenticia y mortalidad. Con respecto a los resultados globales de peso vivo se encontró diferencias estadísticamente significativas $(\mathrm{P}<0.05)$ entre lotes. Aunque no se observó diferencias $(\mathrm{P}>0.05)$ entre lotes al día de edad. En relación a la ganancia de peso en todo el período de estudio, se halló diferencias $(\mathrm{P}<0.05)$, entre lotes siendo mayor en el L1 que L2 y L3.

Cuadro 1 Parámetros productivos de codornices japonesas de tres planteles de reproductores.

\begin{tabular}{lrrr}
\hline Índice & L1 & L2 & \multicolumn{1}{c}{ L3 } \\
\hline Peso vivo inicial, g & $8.1^{\mathrm{a}^{\mathrm{a}}}$ & $8.3^{\mathrm{a}}$ & $8.2^{\mathrm{a}}$ \\
Peso vivo final, g & $114.3^{\mathrm{a}}$ & $113.8^{\mathrm{b}}$ & $108.6^{\mathrm{c}}$ \\
Ganancia de peso acumulado, g & $106.2^{\mathrm{a}}$ & $105.5^{\mathrm{b}}$ & $100.4^{\mathrm{c}}$ \\
Consumo de alimento acumulado, g & $309.3^{\mathrm{a}}$ & $309.1^{\mathrm{a}}$ & $305.6^{\mathrm{b}}$ \\
ICA acumulado & $2.71^{\mathrm{a}}$ & $2.72^{\mathrm{a}}$ & $2.8^{\mathrm{b}}$ \\
Mortalidad acumulada, \% & $3.4^{\mathrm{a}}$ & $4.1^{\mathrm{a}}$ & $3.2^{\mathrm{a}}$ \\
\hline
\end{tabular}

* Promedios dentro de la misma fila con letra diferente muestran significancia estadistica $(\mathrm{P}<0.05)$

El consumo de alimento y el ICA fueron diferentes $(\mathrm{P}<0.05)$ entre los $\mathrm{L} 3$ con los L1 y L2 durante las cuatro semanas de crianza (Cuadro 1). Los L1 y L2, no fueron diferentes $(P>0.05)$ para consumo de alimento acumulado y el ICA y fueron similares a lo reportado por Figueroa y Sulca (1997).

La diferencia del peso corporal promedio obtenido por los tres lotes analizados, a pesar de haber estado bajo las mismas 
condiciones de manejo y sanidad, muestran la influencia de los reproductores padres, quienes transfieren el potencial genético a su descendencia. Al respecto, el L 3 se mantiene muy por debajo de los $\mathrm{L} 1$ y 2 .

Los indices de conversión alimenticia acumulado, a las cuatro semanas fueron similares $(\mathrm{P}>0.05)$ entre los L1 y L2 ambos diferentes $(\mathrm{P}<0.05)$ al $\mathrm{L} 3$.

El porcentaje de mortalidad fue similar en los tres lotes $(\mathrm{P}>0.05)$, durante todo el período de crianza (Cuadro 2), notándose que los accidentes son la mayor causa de pérdidas.

Cuadro 2. Causas de mortalidad (\%) de codornices japonesas de tres planteles de reproductores.

\begin{tabular}{lccc}
\hline Causa & L1 & L2 & L3 \\
\hline Ahogado & 52 & 32 & 39 \\
Pisado & 37 & 45 & 23 \\
Retrazo & 5 & 15 & 21 \\
Onfalitis & 6 & 8 & 17 \\
\hline
\end{tabular}

Edgar et al. (1964), señalaron que la codorniz japonesa era sensible a las enfermedades comunes del pollo; pero es una especie muy resistente cuando recibe un manejo adecuado. La mortalidad en el estudio fue mayor durante la primera semana de edad de las codornices, con porcentajes de $2.4,2.8$ y 1.9 , para $L 1, L 2$ y L3, respectivamente, lo cual está dentro del rango considerado como normal. Posteriormente, a partir de la segunda semana de edad la mortalidad disminuyó notoriamente.

Durante el estudio las codornices japonesas lograron un peso vivo promedio de $112.2 \mathrm{~g}$ y una ganancia de peso total de 103 $\mathrm{g}$, lo cual muestra el crecimiento acelerado de estas aves. Consumieron en promedio $308 \mathrm{~g}$ de alimento, logrando un índice de conversión acumulado de 2.74. La mortalidad acumulada fue baja (3.6\%) probablemente debido a la administración preventiva de vitaminas y antibióticos y a la bioseguridad practicada.

\section{Litentura ctwd}

1. Edgar, S.A.; Y. Weggoner y C. Flanagan. 1964. Susceptibility of coturnix quail to certain disease producing carcinoma to poultry. Poultry Sci. 43: 1315.

2. Figueroa E. y P. Sulca. 1997. Desarrollo de la crianza de la codorniz y su evaluación técnico productiva. Scienta Omni. UNMSM. Of. General de Investigación y Planificación. May. Vol 1:3. 